\title{
EGFR expression is associated with decreased benefit from trastuzumab in the NCCTG N9831 (Alliance) trial
}

H Cheng ${ }^{1}, \mathrm{~K} \mathrm{Ballman}^{2}$, M Vassilakopoulou ${ }^{3}, \mathrm{~A}$ C Dueck ${ }^{4}, \mathrm{M} \mathrm{M} \mathrm{Reinholz}{ }^{5}, \mathrm{~K}$ Tenner $^{2}, \mathrm{~J} \mathrm{Gralow}^{6}, \mathrm{C} \mathrm{Hudis}^{7}$, N E Davidson ${ }^{8}$, G Fountzilas ${ }^{9,10}$, A E McCullough ${ }^{11}$, B Chen ${ }^{5}$, A Psyrri $^{10,12}$, D L Rimm ${ }^{1}$ and E A Perez ${ }^{*, 13}$

${ }^{1}$ Department of Pathology, Yale University School of Medicine, 310 Cedar Street BML116, New Haven, CT 06520, USA; ${ }^{2}$ Division of Biomedical Statistics and Informatics, Mayo Clinic, 200 First Street Southwest, Rochester, MN 55905, USA; ${ }^{3}$ Department of Medical Oncology, Pitie-Salpetriere Hospital, 83 Boulevard de I'Hôpital, 75013 Paris, France; ${ }^{4}$ Section of Biostatistics, Mayo Clinic, 13400 East Shea Boulevard, Scottsdale, AZ 85259, USA; ${ }^{5}$ Department of Laboratory Medicine and Pathology, Mayo Clinic, 200 First Street Southwest, Rochester, MN 55905, USA; ${ }^{6}$ Department of Medical Oncology, University of Washington/ Seattle Cancer Care Alliance, 825 Eastlake Avenue East, Seattle, WA 98109, USA; ${ }^{7}$ Department of Medical Oncology, Memorial Sloan-Kettering Cancer Center, 1275 York Avenue, Box 8, New York, NY 10065, USA; ${ }^{8}$ Division of Hematology/Oncology, University of Pittsburgh Cancer Institute and UPMC Cancer Center, 5150 Centre Avenue, Pittsburgh, PA 15232, USA; ${ }^{9}$ Department of Medical Oncology, Papageorgiou General Hospital, Aristotle University of Thessaloniki School of Medicine, Efkarpia Peripheral Road Stavroupoli, 56429 Thessaloniki, Greece; ${ }^{10}$ Hellenic Cooperative Oncology Group (HeCOG), Laskaridou 1, 11524 Athens, Greece; ${ }^{11}$ Anatomic Pathology, Mayo Clinic, 13400 E Shea Blvd, Scottsdale, AZ 85259, USA; ${ }^{12}$ Second Department of Internal Medicine Propaedeutic, Oncology Section, Attikon University Hospital, University of Athens Medical School, 1 Rimini Street, Haidari, 12462 Athens, Greece and ${ }^{13}$ Department of Hematology/Oncology, Mayo Clinic, 4500 San Pablo Road, Jacksonville, FL 32224, USA

Background: Epidermal growth factor receptor (EGFR) has been hypothesised to modulate the effectiveness of anti-HER2 therapy. We used a standardised, quantitative immunofluorescence assay and a novel EGFR antibody to evaluate the correlation between EGFR expression and clinical outcome in the North Central Cancer Treatment Group (NCCTG) N9831 trial.

Methods: Tissue microarrays were constructed that allowed analysis of 1365 patients randomly assigned to receive chemotherapy alone (Arm A), sequential trastuzumab after chemotherapy (Arm B) and chemotherapy with concurrent trastuzumab (Arm C). Measurement of EGFR was performed using the EGFR antibody, D38B1, on the fluorescence-based AQUA platform. The result was validated using an independent retrospective metastatic breast cancer cohort $(n=130)$.

Results: Epidermal growth factor receptor assessed as a continuous (logarithmic transformed) variable shows an association with disease-free survival in Arm C ( $P=0.009)$ but not in Arm A or B. High EGFR expression was associated with worse outcome (Hazard ratio $(H R)=2.15 ; 95 \% \mathrm{Cl} 1.28-3.60, P=0.004)$. Validation in a Greek metastatic breast cancer cohort showed an $\mathrm{HR}$ associated with high EGFR expression of $1.92(P=0.0073)$.

Conclusions: High expression of EGFR appears to be associated with decreased benefit from adjuvant concurrent trastuzumab. Since other treatment options exist for HER2-driven tumours, further validation of these data may select patients for alternative or additive therapy.

*Correspondence: Dr EA Perez; E-mail: perez.edith@mayo.edu

Received 18 December 2013; revised 23 June 2014; accepted 13 July 2014; published online 12 August 2014

(c) 2014 Cancer Research UK. All rights reserved 0007-0920/14 
Trastuzumab is a humanised monoclonal antibody against the extracellular domain of human epidermal growth factor receptor-2 (HER2) and has shown therapeutic benefit in the 15-20\% of breast cancer patients who overexpress HER2 with impressive improvements in clinical benefit and survival when added to chemotherapy in the metastatic setting (Vogel et al, 2002; Baselga et al, 2010; Perez and Spano, 2012). When added to chemotherapy in the adjuvant setting, disease-free survival (DFS) at 4 years has been demonstrated at $85.7 \%$, compared with $73.7 \%$ in the control arm (Perez et al, 2011a,b; Romond et al, 2012). Despite significant improvement in clinical outcome of patients with HER2-positive disease, not all patients benefit from trastuzumab. In the metastatic setting, 50\% of patients do not achieve $>50 \%$ tumour shrinkage, representing de novo drug resistance even when combined with chemotherapy (Zito et al, 2012). As the number of therapeutic options increase for those with HER2 overexpressing breast cancer, patients would benefit from new biomarkers to increase specificity of companion diagnostic tests to better predict benefit for specific anti-HER2 therapies.

Human epidermal growth factor receptor 1 (EGFR) is a key member of the HER signalling pathway that may be associated with trastuzumab resistance. Cell line models have revealed that overexpression and activation of EGFR induces trastuzumab resistance in HER2-positive cell lines (Kumar, 2007; Dua et al, 2010). However, data supporting EGFR assessment as a biomarker of trastuzumab resistance in breast cancer patients remain inconclusive. Perhaps the most critical variable preventing common usage of EGFR as a biomarker is non-reproducibility of its assessment. Many antibodies have been generated for EGFR assessment, but most require a protease treatment in the process of antigen retrieval for immunohistochemistry (IHC). The lack of reproducibility associated with this issue and poor agreement between different commercially available EGFR antibodies (Anagnostou et al, 2010) have resulted in confusing literature on assessment of the impact of EGFR testing and lack of its adoption as a companion diagnostic test. Recently, a new monoclonal antibody for EGFR, D38B1, has been developed against the cytoplasmic domain of the molecule and does not require protease treatment for antigen retrieval. Using this antibody, our group has standardised a sensitive, specific, and reproducible fluorescencebased, quantitative EGFR assay (Dimou et al, 2011). In this study, we used this assay to evaluate the association between EGFR expression and trastuzumab response in the North Central Cancer Treatment Group (NCCTG) N9831 trial. We then validated the result in a retrospectively collected trastuzumab-treated metastatic breast cancer cohort from Greece.

The NCCTG (now Alliance) N9831 trial assessed the efficacy and safety of adding 52 weeks of trastuzumab to the standard anthracycline/taxane-based chemotherapy arm (Perez et al, 2011a). It enrolled 3505 patients, randomly assigned to chemotherapy plus trastuzumab regimes and a control chemotherapy treatment regimen. The trial was among four large clinical trials that led to FDA and global approval of the usage of trastuzumab in the adjuvant setting. In the study reported herein, a tissue microarray (TMA) containing 1444 samples in three-fold redundancy representing 1365 patients (1231 evaluable and 134 failed) from this trial was assessed for EGFR expression using the recently described standardised EGFR assay. Our goal was to determine whether EGFR expression was associated with benefit from trastuzumab in the adjuvant setting.

\section{MATERIALS AND METHODS}

Cohort description. The NCCTG N9831 trial is a three-arm prospective phase III randomised trial evaluating adjuvant therapy for patients with HER2-positive resected early stage breast cancer (Perez et al, 2011b, 2012). Eligible patients were randomly assigned into three arms. Arm A is a control arm, in which patients received AC followed by weekly paclitaxel; Arm B is a sequential arm, in which patients received AC followed by weekly paclitaxel followed by trastuzumab; and Arm C is a concurrent arm, in which patients received AC followed by weekly paclitaxel plus trastuzumab followed by trastuzumab alone. Patients were enrolled as a collaborative effort between NCCTG and other NCIsponsored cancer cooperative groups (ECOG, SWOG, and CALGB).

N9831 enrolled 3505 women, of whom 2823 provided consent and were deemed eligible upon central review. For this study, there were 1231 women in the TMA for evaluation (403 from Arm A, 452 from Arm B, and 376 from Arm C; Supplementary Figure 1) and 1592 women not present in the TMA: 1458 due to inadequate tissue (513 Arm A, 478 Arm B, and 467 Arm C) and 134 due to tissue loss in the TMA (46 Arm A, 53 Arm B, and 35 Arm C). Of the 1231 women with measurements, 274 women had one measurement, 434 had two measurements, 470 women had three measurements, and 53 women had measurements from more than one block. Average scores were used to represent each patient.

A retrospectively collected cohort of metastatic breast cancer patients treated with various chemotherapy regimens with trastuzumab was obtained collaboratively from the Hellenic Cooperative Oncology Group (HeCOG), Athens, Greece. The TMAs contained 149 patients with up to four-fold redundancy. Of the 149 patients, 130 patients received trastuzumab in first-line treatment and were included in the analysis. Of these 130 patients, four had four measurements, 4 had three measurements, 65 had two measurements, and 35 had one measurement. Average scores were used to represent each patient. All measurements were made on tissue from the primary tumour. The detailed cohort descriptions are shown in Supplementary Tables 1 and 2. More detailed description of this cohort is given in a publication by Razis et al (2011).

Quantitative immunofluorescence-based assessment of EGFR. Tissue microarray slides were prepared at Mayo Clinic and shipped to Yale (DR's laboratory) for analysis. After deparaffinisation, antigen retrieval was performed using a PT module (LabVision Corp., Fremont, CA, USA) with EDTA buffer $\left(\mathrm{pH} 8,97^{\circ} \mathrm{C}\right.$; $20 \mathrm{~min}$ ). Slides were then incubated in $2.5 \%$ hydrogen peroxide ( $30 \mathrm{~min}$; room temperature) to block endogenous peroxidase activity. The following steps were performed in an autostainer (LabVision Corp.). Non-specific antigens were blocked via incubation in $0.3 \%$ bovine serum albumin in Tris-buffered saline solution and Tween-20 (30 min; room temperature). Slides were then incubated overnight with a cocktail of a primary EGFR antibody clone D38B1 (1:200; Cell Signaling Technology, Danvers, MA, USA) and a mouse monoclonal cytokeratin antibody (Dako Corp., Carpinteria, CA, USA). Next, a cocktail of Alexa 546conjugated goat anti-mouse secondary antibody (Molecular Probes, Inc., Eugene, OR, USA) diluted 1:100 in rabbit EnVision reagent (Dako Corp.) was applied to the slides ( $1 \mathrm{~h}$; room temperature). Cyanine 5-tyramide was used to develop the fluorescent signal $(1: 50$, room temperature, 10 min Perkin-Elmer, Inc., Waltham, MA, USA). Finally, Prolong Gold (Molecular Probes, Inc.) containing DAPI was used to detect nuclei. An index array was stained aside each cohort array to enable standardisation of the assay, along with negative (no primary) and positive controls.

The AQUA method of quantitative immunofluorescence (QIF) was used to quantitatively measure EGFR expression as previously described in lung cancer (Zito et al, 2012) and other reviews (Camp et al, 2002; Gustavson et al, 2009). The AQUA scores of EGFR in 
the tumour mask were calculated as the sum of the target EGFR intensity in the tumour mask divided by the area of the tumour mask. The AQUA scores were normalised for exposure time, bit depth and lamp hours for optimal standardisation and reproducibility.

To calculate the absolute EGFR level in the cell, two steps of normalisation were applied. The AQUA score of EGFR measured in the N9831 cohort was first normalised to a standardised index array, which consists of a series of lung cancer cases and cell lines and was previously used to define EGFR concentrations in a lung cancer study (Dimou et al, 2011). The normalised AQUA score was translated into concentration units of EGFR (nanograms of EGFR per microgram of total protein), using the standard curve derived from the previous study (Dimou et al, 2011).

Statistical analysis. Statistical calculations were performed using SAS version 9.3 (SAS, Cary, NC, USA) and R version 2.14.2 (The R Foundation for Statistical Computing). The data for these analyses were locked on 13 July 2011. The end point in the adjuvant N9831 cohort analysis was DFS, defined as time from random assignment to documentation of any evidence of recurrence of breast cancer; contralateral breast cancer; second primary cancer; or death resulting from any cause. Patients were censored at time of last follow-up if they had not had an event of interest. Differences between groups were compared using a chi-square test (for categorical variables) or a two-sample t-test (for continuous variables). Univariable and multivariable Cox proportional hazard models were used to determine associations between EGFR values and DFS. The EGFR distribution was extremely skewed (Supplementary Figure 2) and so the (natural) logarithm of the EGFR value (lnEGFR) was used in the analysis. Given the skewed nature of the EGFR values, it was felt that EGFR would likely have a threshold effect. The optimal cutoff point of continuous EGFR into low and high values was determined using recursive partitioning (rpart in $\mathrm{R}$ ) for Arm $\mathrm{C}$ patients in the NCCTG N9831 cohort. Arm C alone was used to define the optimal cutoff

A
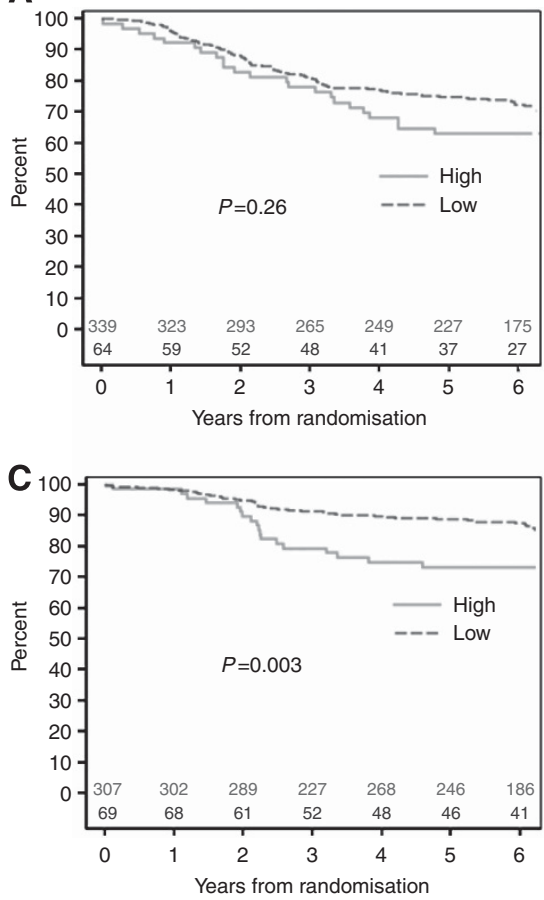

value because it was the arm that exhibited from the most benefit of chemotherapy and trastuzumab treatment and we were interested in the association of EGFR with trastuzumab benefit. The outcome value for the rpart analysis was DFS and EGFR level was the only explanatory variable. Since rpart does not require variables be normally distributed, the original EGFR values (i.e., not log transformed) were used. The selected threshold was EGFR level $=13 \mathrm{ng} \mu \mathrm{g}^{-1}$. The same cutoff point was applied to Arms A and $B$ and survival differences between high and low EGFR groups were estimated using Kaplan-Meier (KM) estimates and compared with a log-rank test. Stratification factors for the N9831 trial (age at trial registration, extent of surgery, hormone receptor status, tumour size, and number of positive nodes) were all used in the multivariable Cox model. The Greek cohort of women with metastatic breast cancer was used as a validation cohort with an end point of progression-free survival, which was defined as the time from initiation of trastuzumab as a first-line treatment to first report of patient disease progression or death. All statistical tests were two-sided and significance level was $P=0.05$.

\section{RESULTS}

The median follow-up time of the 1231 patients evaluable for EGFR was 7.0 years. A comparison of the 1231 women with an EGFR measurement to the 2274 without an EGFR measurement revealed some statistically significant differences between the groups (Supplementary Table 1). On average, women with EGFR analysis were more likely to have axillary node dissection $(92 \%$ vs $89 \%$ ), more lymph-node involvement ( $88 \%$ vs $86 \%$ with one or more node involved), and larger tumours (64\% vs 59\% with tumours $>2.0 \mathrm{~cm}$ ), and less likely to be oestrogen receptor positive compared to women without EGFR analysis (49\% vs 54\%). Many of these differences reflect the fact that women with more disease were more likely to have greater amounts of tissue available for TMA production and correlative studies.

B

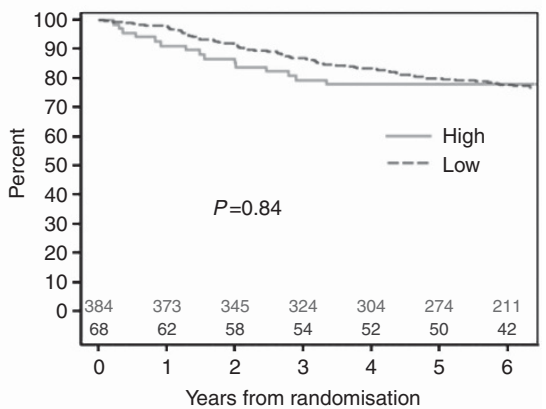

Figure 1. (A-C) DFS comparison of EGFR high group vs EGFR not high in the N9831 cohort, stratified by treatment arm. (A) Arm A patients: Kaplan-Meier curves for low EGFR patients and high EGFR patients. (B) Arm B patients: Kaplan-Meier curves for low EGFR patients and high EGFR patients. (C) Arm C patients: Kaplan-Meier curves for low EGFR patients and high EGFR patients. 
Association of InEGFR and DFS in the NCCTG N9831 cohort. The logarithm transformed EGFR values were univariably associated with DFS in Arm C $(P$-value $=0.009)$ but not in Arm A $(P$-value $=0.31)$ or Arm B $(P$-value $=0.93)$. When the arms were pooled $\operatorname{lnEGFR}$ was not associated with DFS in the univariable model $(P$-value $=0.072)$ and was not associated with DFS in the multivariable model that adjusted for the trial stratification variables $(P$-value $=0.31)$.

High expression of EGFR is associated with worse survival outcome in the NCCTG N9831 cohort. The cutpoint for EGFR determined by rpart was $13 \mathrm{ng} \mu \mathrm{g}^{-1}$. Women with an EGFR AQUA score of above $13 \mathrm{ng} \mu \mathrm{g}^{-1}$ were classified as the EGFR high group; there were 201 out of 1231 women in this group (16.3\%): 64 (15.9\%) in Arm A, 68 (15.0\%) in Arm B, and 69 (18.4\%) in Arm C. Although women with high EGFR in Arms A and B had slightly lower DFS compared to women with low EGFR, the differences were not statistically significant (Figure 1A and B). In Arm C, women with high EGFR had a significantly lower DFS compared to women with low EGFR (Figure 1C). The percent of women with high EGFR who were disease free at 5 years is $73.2 \%$ compared with $88.8 \%$ of women with low EGFR $(P$-value $=0.003)$. Survival curves were also constructed to compare arms of the trial within the low (Figure 2A) and high (Figure 2B) EGFR groups. Patients with low EGFR show stratification by treatment arm similar to that observed in the original trial. Patients with high EGFR showed no benefit in the trastuzumab arms compared with the control arm.
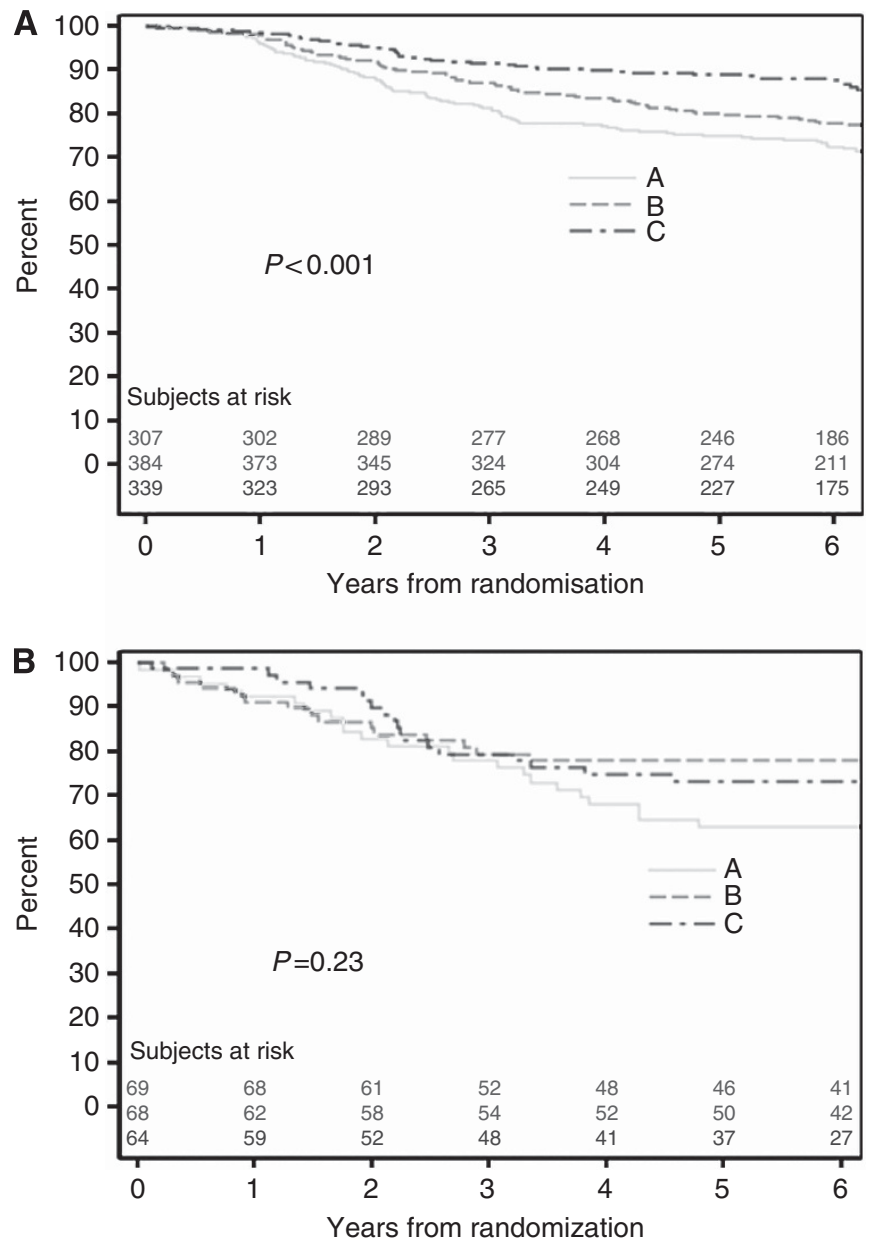

Figure 2. (A, B) Survival curves for each trial arm in N9831 patients with low (A) or high (B) EGFR levels.
The association between EGFR status (high vs low) and DFS was evaluated with univariable and multivariable models (Table 1) for each of the three treatment arms. The association was statistically significant for Arm $\mathrm{C}$ in both univariable and multivariable models, but not for Arms A and B in either model. Multivariable analysis of all collected clinical variables within Arm C shows that EGFR was an independent predictive variable (Table 2). Notably, EGFR was associated with DFS when adjusting for oestrogen and progesterone receptor status. A test for interaction between EGFR status and the treatment arms (Arm A vs Arm C) did not achieve statistical significance in both the univariable model $(P$-value $=0.15)$ and the multivariable model $(P$-value $=0.15)$, which could be due to the limited power to detect an interaction.

Validation in the Greek cohort. Although the univariable analysis revealed a continuous relationship between $\operatorname{lnEGFR}$ and DFS in Arm C, the data were dichotomised on the original scale due to the extremely skewed nature of the EGFR values and the thought it was likely to have a threshold effect; this also would classify patients for future studies as positive or negative. The N9831 data were used to define the value of $13 \mathrm{ng} \mu \mathrm{g}^{-1}$ as the cutpoint on the original EGFR scale. To validate the cutpoint and determine whether EGFR was truly predictive $v s$ prognostic, the

Table 1. Cox proportional hazard models evaluating the association between EGFR group and DFS

\begin{tabular}{|c|c|c|c|c|}
\hline & \multicolumn{2}{|c|}{ Univariable model } & \multicolumn{2}{|c|}{ Multivariable model } \\
\hline & HR (95\% Cl) & $P$-value & HR $(95 \% \mathrm{Cl})$ & $P$-value \\
\hline Arm A & $1.29(0.82-2.04)$ & 0.26 & $1.18(0.74-1.89)$ & 0.49 \\
\hline Arm B & $0.95(0.55-1.63)$ & 0.84 & $0.91(0.52-1.61)$ & 0.75 \\
\hline Arm C & $2.15(1.28-3.60)$ & 0.004 & $1.83(1.05-3.17)$ & 0.03 \\
\hline \multicolumn{5}{|c|}{$\begin{array}{l}\text { Abbreviations: } \mathrm{Cl}=\text { confidence interval; } D F S=\text { disease-free survival; } E G F R=\text { epidermal } \\
\text { growth factor receptor; } H R=\text { Hazard ratio. }\end{array}$} \\
\hline
\end{tabular}

\begin{tabular}{|c|c|c|c|c|}
\hline Variable & $\begin{array}{c}\text { Hazard } \\
\text { ratio }\end{array}$ & $\begin{array}{l}95 \% \text { confidence } \\
\text { interval }\end{array}$ & Chi-Sq & $P$ \\
\hline \multicolumn{5}{|l|}{ Age group } \\
\hline$\leqslant 50$ vs $<50$ & 1.57 & $0.952-2.580$ & 3.122 & 0.077 \\
\hline \multicolumn{5}{|l|}{ Grade } \\
\hline III vs I and II & 1.77 & $0.950-3.283$ & 3.233 & 0.072 \\
\hline \multicolumn{5}{|l|}{ ER } \\
\hline Positive vs negative & 0.76 & $0.439-1.327$ & 0.917 & 0.338 \\
\hline \multicolumn{5}{|l|}{ Nodal status } \\
\hline Negative vs positive & 0.72 & $0.334-1.554$ & 0.699 & 0.403 \\
\hline \multicolumn{5}{|l|}{ EGFR } \\
\hline High vs low & 1.83 & $1.048-3.176$ & 4.523 & 0.033 \\
\hline
\end{tabular}


ideal data set would be one where some HER2-positive women were treated with chemotherapy alone and others were treated with chemotherapy plus trastuzumab. Unfortunately, we did not have access to such a cohort of women. Instead a metastatic cohort was available from a small multi-institutional trial in Greece. The cohort contained 130 patients treated with first-line trastuzumab. Continuous assessment of EGFR using the Cox proportional hazard model, with PFS as the end point, was statistically significant $(P=0.0366)$. When EGFR was dichotomised at $13 \mathrm{ng} \mu \mathrm{g}^{-1}$ and assessed by $\mathrm{KM}$ analysis, the median difference in PFS was 6.1 months (log-rank $P=0.0063$ ) (Figure 3). The univariable binary HR is 1.92 (95\% CI: $1.192-3.092, P=0.0073$ ). The association between EGFR and PFS remained significant when age, grade, distant metastasis, ER, and HER2 status were controlled (Table 3).

\section{DISCUSSION}

Although EGFR expression has been associated with trastuzumab resistance in cell line models (Kumar, 2007; Dua et al, 2010), previous biomarker studies of EGFR had not validated this observation in human tumours (Gori et al, 2009). In part, this could be due to challenges associated with measurement of EGFR by traditional IHC. Initially, the EGFR assay was established as a companion diagnostic assay with early trials of cetuximab in colon cancer (Goldstein and Armin, 2001). However, the assay was not standardised and often $75 \%$ or more were judged as positive (Goldstein and Armin, 2001; Heinemann et al, 2009). A study of EGFR in breast cancer showed a much lower rate $(<10 \%)$ of EGFR overexpression in breast cancer (Bhargava et al, 2005). Ultimately, studies showed that EGFR status by IHC made no difference in outcome of colon cancer patients (Lenz et al, 2006) and CAP surveys of laboratories were unable to reach consensus, so the assay was largely discarded. The root of the problem was most likely the challenge of standardisation of the EGFR assay. Assessment of EGFR using antibodies from varied vendors or using varied assay conditions can produce widely variable results (Anagnostou et al, 2010). A portion of that variability was likely due to the requirement for protease-based antigen retrieval for many antibodies. This step is highly variable and difficult to standardise. A second issue may be that different laboratories used antibodies targeting different regions of the protein. Here, we used a newly commercialised EGFR antibody (D38B1) that does not require protease-based antigen retrieval and has been previously standardised to exact protein concentrations (Dimou et al, 2011).

Patients with high EGFR did not appear to benefit from concurrent trastuzumab or sequential trastuzumab whereas those with low EGFR values experienced benefits similar to those observed in the parent trial. One explanation for this observation could be that only tumours that are strongly driven by HER2 are affected by trastuzumab and when higher levels of heterodimer are present due to high EGFR, the effect of trastuzumab is attenuated. In a lung cancer model system, chemotherapy was associated with activation of the EGFR pathway as shown by phosphorylation of EGFR (Van Schaeybroeck et al, 2006). It may be that both the increased level of the receptor and its activation by chemotherapy combine to evade effects of trastuzumab.

The QIF method used here is a continuous method, but treatment decisions are binary. Thus to be useful as a companion diagnostic test and due to the skewed nature of the EGFR distribution, EGFR was dichotomised to better direct therapy. Since there is no known biological cutpoint indicating the level at which EGFR exerts its effect, we used rpart to discover the most meaningful cutpoint for use in future studies. We were unable to access a perfectly matched adjuvant cohort, but were able to test a

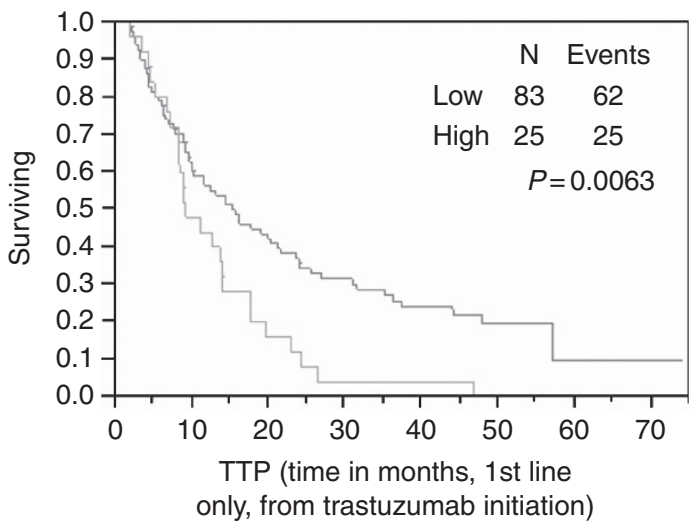

Figure 3. Patients with high level of EGFR (blue line) as defined by the cutpoint from the $\mathrm{N} 9831$ cohort show less benefit from trastuzumab treatment in the Greek metastatic breast cancer cohort. The red line shows the 83 patients above the cutpoint and the blue line shows those below. A full color version of this figure is available at the British Journal of Cancer journal online.

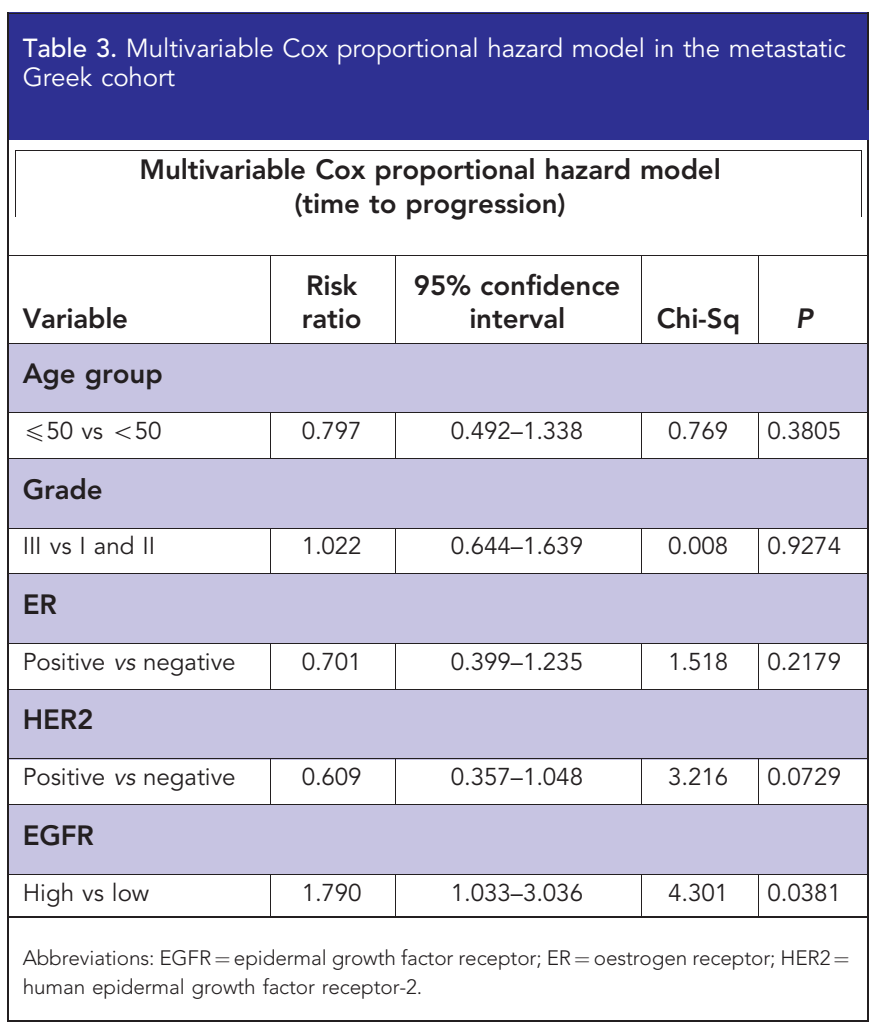

retrospective metastatic cohort which validated the discovered cutpoint. To further validate this cutpoint and EGFR as a predictive $v s$ prognostic biomarker, we are pursuing efforts to obtain tissue from other adjuvant trastuzumab trials.

Additional potential limitations exist for this study. Perhaps most significant is that this work was performed entirely on TMAs. While the data were largely derived from the average of two or three spots, the assay has not yet been evaluated on whole slide specimens, as would occur in a true clinical setting. A second weakness is the retrospective nature of the work. Although the examination of the effect of EGFR was built into the prospective study design of NCCTG N9831, the examination was to be correlative and at the time of the trial design, the quantitative 
approach used here was not anticipated. Nonetheless, this type of prospectively designed retrospective analysis is still considered as an acceptable means of obtaining level 2 evidence for the value of a biomarker (Simon et al, 2009). Finally, the fact that the validation set is a metastatic cohort, rather than adjuvant, and the fact that there were a high number of discordant (local vs central HER2 score) cases all weaken the conclusions. Given these weaknesses, our correlative/translational data should be interpreted as hypothesis generating.

In spite of the above limitations, we feel the observation is still compelling because of the mechanistic relationship between EGFR and HER2 and because of the availability of the dual inhibitor lapatinib (Xia et al, 2002; Scaltriti et al, 2009). While the ALTTO trial is still underway (http://clinicaltrials.gov/ct2/show/ NCT00490139), the Neo-ALTTO trial showed benefit in both the trastuzumab and lapatinib arms, with maximal benefit seen in the combination arm. Our study suggests that EGFR expression should be measured on the patients in that trial, and we plan to do so. We hypothesise that the subset of patients with high EGFR expression may show better response in the lapatinib or combination arms compared with the arm of those treated with trastuzumab alone. Though future studies are needed before moving this assay to the clinic, this work provides provocative preliminary data suggesting that EGFR measurement may provide a method for personalising treatment in breast cancer, beyond the single assay for HER2.

\section{ACKNOWLEDGEMENTS}

This study was supported by NIH RO1 CA-139431 (DLR) and CA-129949 (EAP), U10 CA25224 (EAP). Preliminary reports from this study were presented at the Thirty-Fifth Annual CTRC-AACR San Antonio Breast Cancer Symposium 3-7 December 2012; San Antonio, TX (abstract 850544).

\section{CONFLICT OF INTEREST}

Dr Gralow receives research funding from Amgen, Novartis, Roche, and Genentech. Dr Hudis receives research funding from Merck. Dr Rimm is a consultant for Genoptix/Novartis, the licensee of the Yale-owned AQUA technology used in this study. The remaining authors declare no conflict of interest.

\section{REFERENCES}

Anagnostou VK, Welsh AW, Giltnane JM, Siddiqui S, Liceaga C, Gustavson M, Syrigos KN, Reiter JL, Rimm DL (2010) Analytic variability in immunohistochemistry biomarker studies. Cancer Epidemiol Biomarkers Prev 19(4): 982-991.

Baselga J, Gelmon KA, Verma S, Wardley A, Conte P, Miles D, Bianchi G, Cortes J, McNally VA, Ross GA, Fumoleau P, Gianni L (2010) Phase II trial of pertuzumab and trastuzumab in patients with human epidermal growth factor receptor 2-positive metastatic breast cancer that progressed during prior trastuzumab therapy. J Clin Oncol 28(7): 1138-1144.

Bhargava R, Gerald WL, Li AR, Pan Q, Lal P, Ladanyi M, Chen B (2005) EGFR gene amplification in breast cancer: correlation with epidermal growth factor receptor mRNA and protein expression and HER-2 status and absence of EGFR-activating mutations. Mod Pathol 18(8): 1027-1033.

Camp RL, Chung GG, Rimm DL (2002) Automated subcellular localization and quantification of protein expression in tissue microarrays. Nat Med 8(11): 1323-1327.

Dimou A, Agarwal S, Anagnostou V, Viray H, Christensen S, Gould Rothberg B, Zolota V, Syrigos K, Rimm DL (2011) Standardization of epidermal growth factor receptor (EGFR) measurement by quantitative immunofluorescence and impact on antibody-based mutation detection in non-small cell lung cancer. Am J Pathol 179(2): 580-589.
Dua R, Zhang J, Nhonthachit P, Penuel E, Petropoulos C, Parry G (2010) EGFR over-expression and activation in high HER2, ER negative breast cancer cell line induces trastuzumab resistance. Breast Cancer Res Treat 122(3): 685-697.

Goldstein NS, Armin M (2001) Epidermal growth factor receptor immunohistochemical reactivity in patients with American Joint Committee on Cancer Stage IV colon adenocarcinoma: implications for a standardized scoring system. Cancer 92(5): 1331-1346.

Gori S, Sidoni A, Colozza M, Ferri I, Mameli MG, Fenocchio D, Stocchi L, Foglietta J, Ludovini V, Minenza E, De Angelis V, Crino L (2009) EGFR, pMAPK, pAkt and PTEN status by immunohistochemistry: correlation with clinical outcome in HER2-positive metastatic breast cancer patients treated with trastuzumab. Ann Oncol 20(4): 648-654.

Gustavson MD, Bourke-Martin B, Reilly DM, Cregger M, Williams C, Tedeschi G, Pinard R, Christiansen J (2009) Development of an unsupervised pixel-based clustering algorithm for compartmentalization of immunohistochemical expression using Automated QUantitative Analysis. Appl Immunohistochem Mol Morphol 17(4): 329-337.

Heinemann V, Stintzing S, Kirchner T, Boeck S, Jung A (2009) Clinical relevance of EGFR- and KRAS-status in colorectal cancer patients treated with monoclonal antibodies directed against the EGFR. Cancer Treat Rev 35(3): 262-271.

Kumar R (2007) ErbB-dependent signaling as a determinant of trastuzumab resistance. Clin Cancer Res 13(16): 4657-4659.

Lenz HJ, Van Cutsem E, Khambata-Ford S, Mayer RJ, Gold P, Stella P, Mirtsching B, Cohn AL, Pippas AW, Azarnia N, Tsuchihashi Z, Mauro DJ, Rowinsky EK (2006) Multicenter phase II and translational study of cetuximab in metastatic colorectal carcinoma refractory to irinotecan, oxaliplatin, and fluoropyrimidines. J Clin Oncol 24(30): 4914-4921.

Perez EA, Dueck AC, McCullough AE, Reinholz MM, Tenner KS, Davidson NE, Gralow J, Harris LN, Kutteh LA, Hillman DW, Jenkins RB, Chen B (2012) Predictability of adjuvant trastuzumab benefit in N9831 patients using the ASCO/CAP HER2-positivity criteria. J Natl Cancer Inst 104(2): 159-162.

Perez EA, Romond EH, Suman VJ, Jeong JH, Davidson NE, Geyer Jr CE, Martino S, Mamounas EP, Kaufman PA, Wolmark N (2011a) Four-year follow-up of trastuzumab plus adjuvant chemotherapy for operable human epidermal growth factor receptor 2-positive breast cancer: joint analysis of data from NCCTG N9831 and NSABP B-31. J Clin Oncol 29(25): 3366-3373.

Perez EA, Spano JP (2012) Current and emerging targeted therapies for metastatic breast cancer. Cancer 118(12): 3014-3025.

Perez EA, Suman VJ, Davidson NE, Gralow JR, Kaufman PA, Visscher DW, Chen B, Ingle JN, Dakhil SR, Zujewski J, Moreno-Aspitia A, Pisansky TM, Jenkins RB (2011b) Sequential versus concurrent trastuzumab in adjuvant chemotherapy for breast cancer. J Clin Oncol 29(34): 4491-4497.

Razis E, Bobos M, Kotoula V, Eleftheraki AG, Kalofonos HP, Pavlakis K, Papakostas P, Aravantinos G, Rigakos G, Efstratiou I, Petraki K, Bafaloukos D, Kostopoulos I, Pectasides D, Kalogeras KT, Skarlos D, Fountzilas G (2011) Evaluation of the association of PIK3CA mutations and PTEN loss with efficacy of trastuzumab therapy in metastatic breast cancer. Breast Cancer Res Treat 128(2): 447-456.

Romond EH, Suman VJ, Jeong J, Sledge G, Geyer Jr CE, Martino S, Rastogi P, Gralow J, Swain SM, Winer E, Colon-Otero G, Hudis C, Paik S, Davidson NE, Mamounas EP, Zujewski J, Wolmark N, Perez EA (2012) Trastuzumab plus adjuvant chemotherapy for HER2-positive breast cancer: final planned joint analysis of overall survival (OS) from NSABP B-31 and NCCTG N9831. Cancer Res 72(24_suppl): 105s.

Scaltriti M, Verma C, Guzman M, Jimenez J, Parra JL, Pedersen K, Smith DJ, Landolfi S, Ramon y Cajal S, Arribas J, Baselga J (2009) Lapatinib, a HER2 tyrosine kinase inhibitor, induces stabilization and accumulation of HER2 and potentiates trastuzumab-dependent cell cytotoxicity. Oncogene 28(6): 803-814.

Simon RM, Paik S, Hayes DF (2009) Use of archived specimens in evaluation of prognostic and predictive biomarkers. J Natl Cancer Inst 101(21): 1446-1452.

Van Schaeybroeck S, Kyula J, Kelly DM, Karaiskou-McCaul A, Stokesberry SA, Van Cutsem E, Longley DB, Johnston PG (2006) Chemotherapy-induced epidermal growth factor receptor activation determines response to combined gefitinib/chemotherapy treatment in non-small cell lung cancer cells. Mol Cancer Ther 5(5): 1154-1165. 
Vogel CL, Cobleigh MA, Tripathy D, Gutheil JC, Harris LN, Fehrenbacher L, Slamon DJ, Murphy M, Novotny WF, Burchmore M, Shak S, Stewart SJ, Press M (2002) Efficacy and safety of trastuzumab as a single agent in firstline treatment of HER2-overexpressing metastatic breast cancer. J Clin Oncol 20(3): 719-726.

Xia L, Wang L, Chung AS, Ivanov SS, Ling MY, Dragoi AM, Platt A, Gilmer TM, Fu XY, Chin YE (2002) Identification of both positive and negative domains within the epidermal growth factor receptor $\mathrm{COOH}$-terminal region for signal transducer and activator of transcription (STAT) activation. J Biol Chem 277(34): 30716-30723.
Zito CR, Jilaveanu LB, Anagnostou V, Rimm D, Bepler G, Maira SM, Hackl W, Camp R, Kluger HM, Chao HH (2012) Multi-level targeting of the phosphatidylinositol-3-kinase pathway in non-small cell lung cancer cells. PLoS One 7(2): e31331.

This work is published under the standard license to publish agreement. After 12 months the work will become freely available and the license terms will switch to a Creative Commons AttributionNonCommercial-Share Alike 3.0 Unported License.

Supplementary Information accompanies this paper on British Journal of Cancer website (http://www.nature.com/bjc) 